\title{
Stability of fluxon motion in long Josephson junctions at high bias
}

Pagano, S.; Sørensen, Mads Peter; Christiansen, Peter Leth; Parmentier, R. D.

Published in:

Physical Review B

Link to article, DOI:

10.1103/PhysRevB.38.4677

Publication date:

1988

Document Version

Publisher's PDF, also known as Version of record

Link back to DTU Orbit

Citation (APA):

Pagano, S., Sørensen, M. P., Christiansen, P. L., \& Parmentier, R. D. (1988). Stability of fluxon motion in long Josephson junctions at high bias. Physical Review B, 38(7), 4677-4687.

https://doi.org/10.1103/PhysRevB.38.4677

\section{General rights}

Copyright and moral rights for the publications made accessible in the public portal are retained by the authors and/or other copyright owners and it is a condition of accessing publications that users recognise and abide by the legal requirements associated with these rights.

- Users may download and print one copy of any publication from the public portal for the purpose of private study or research.

- You may not further distribute the material or use it for any profit-making activity or commercial gain

- You may freely distribute the URL identifying the publication in the public portal

If you believe that this document breaches copyright please contact us providing details, and we will remove access to the work immediately and investigate your claim. 


\title{
Stability of fluxon motion in long Josephson junctions at high bias
}

\author{
S. Pagano, ${ }^{*}$ M. P. Soerensen, P. L. Christiansen, and R. D. Parmentier ${ }^{\dagger}$ \\ Laboratory of Applied Mathematical Physics, The Technical University of Denmark, DK-2800 Lyngby, Denmark
}

(Received 29 September 1987; revised manuscript received 16 March 1988)

\begin{abstract}
In long Josephson junctions the motion of fluxons is revealed by the existence of current steps, zero-field steps, in the current-voltage characteristics. In this paper we investigate the stability of the fluxon motion when high values of the current bias are involved. The investigation is carried on by numerical integration of the model equation, the perturbed sine-Gordon equation, simulating junctions of overlap and annular geometry. A detailed description of the mechanism for the switching from the top of the zero-field step for both geometries is reported. Moreover, the effect of the various dissipations and of the junction length on the switching-current value is investigated. A simple boundary model is able to describe, for junctions of overlap geometry, the qualitative dependence of the switching current on the system parameters.
\end{abstract}

\section{INTRODUCTION}

It is well known that Josephson junctions can support, under appropriate conditions, the motion of magnetic field quanta (fluxons). The evidence of such motion is observed experimentally as current singularities [zero-field steps (ZFS's)] in the current-voltage characteristic and as microwave emission, in junctions having physical lengths bigger than the Josephson penetration length $\lambda_{j}{ }^{1}$ Since 1973, an attempt has been made to explain the observed phenomena in terms of oscillatory solitonic solutions of the "perturbed" sine-Gordon equation (PSGE) describing the electrodynamic of a Josephson junction. ${ }^{2}$ For a unidimensional geometry and in normalized units it has the form

$$
\phi_{x x}-\phi_{t t}-\sin \phi=\alpha \phi_{t}-\beta \phi_{x x t}-\gamma .
$$

Here, $\phi$ is the usual Josephson phase variable, $x$ is distance along the junction normalized to the Josephson penetration length $\lambda_{j}, t$ is time normalized to the inverse of the Josephson plasma angular frequency $\omega_{j}, \alpha$ is the normalized shunt conductance that takes into account tunneling of normal electrons across the junction, $\beta$ is the normalized real part of the superconductor surface impedance, $\gamma$ is the dc bias current normalized to the Josephson critical current, and the subscripts indicate partial derivatives. Equation (1), together with the appropriate boundary conditions, determined by the particular junction geometry employed, gives a very good description of the observed dynamical behavior of the junction, often to a surprising degree of accuracy. It has to be noted, however, that since exact analytic dynamical solutions of Eq. (1), are, in general, not known, all the information is obtained either by direct numerical integration of Eq. (1), or by perturbative methods based on the known analytical solutions of the unperturbed sineGordon equation $[\alpha=\beta=\gamma=0$ in Eq. (1)], or by some other approximation scheme.

The perturbative approach is very useful in describing the overall dynamics of fluxons in the junction, in partic- ular when all the perturbing terms are small, but it fails in describing extreme situations such as the ones where the stability of the traveling solutions at high bias values is involved. In fact, the perturbative approach is unable to predict the maximum current amplitude of the ZFS's, i.e., the maximum dc bias current that can sustain fluxon propagation. The simplest power-balance perturbative scheme ${ }^{3}$ predicts an infinite current step height, while the perturbative scheme proposed in Refs. 4 and 5 predicts a maximum normalized step height equal to one. In contrast, experimental and numerical results, by various authors, typically give a maximum step height between 0.4 and $0.8 .^{6}$ The main reason for the failure of the perturbative approach is that, being based on solutions of the pure sine-Gordon equation, it is not valid when the perturbative terms [the right-hand side of Eq. (1)] become large, as occurs when bias values that are not small are considered.

Since the question of the maximum current amplitude of the ZFS's is of considerable practical importance to the experimentalist, and since no completely adequate analytic or perturbative approach is presently available, we propose in this work to furnish a detailed numerical study of the question, with the hope of providing a springboard for future theoretical work. We note that a step in the same direction has recently been taken by Zhang and $\mathrm{Wu}{ }^{7}$ With respect to their work, our work (i) considers junctions of both annular and overlap geometries, ${ }^{1,3}$ (ii) describes in detail the instability mechanism for both geometries, and (iii) employs more than one numerical scheme, which in turn, (iv) pinpoints a numerical pitfall to be avoided. Like Zhang and $\mathrm{Wu}$, we limit attention to dynamic states involving a single propagating fluxon. Moreover, we shall not consider, in this work, the effects of intrinsic and extrinsic noise and of barrier spatial nonuniformities ${ }^{8}$ on the stability of the fluxon oscillations. The reasons for this choice are based on the facts that the cited effects, although often important in physical devices, can always be reduced by a careful shielding of the junction from electromagnetic interference, by lowering the working temperature and by 
improving the fabrication processes. Then the maximum performances will be determined by the intrinsic instabilities of the fluxon oscillations, which are the subject of this work.

In Sec. II a description of the numerical methods used is reported. Junctions of annular geometry and of overlap geometry are then analyzed in order to identify the ranges of stable fluxon motion in each case. Finally, a quasianalytical model is presented which gives reasonable qualitative agreement with the numerical results for overlap geometry junctions.

\section{NUMERICAL METHODS}

In order to numerically solve Eq. (1) with the appropriate boundary condition, two different numerical schemes have been employed. One is based on a Fourier-Galerkin spatial decomposition of the phase $\phi$ as ${ }^{9}$

$$
\begin{array}{r}
\phi(x, t)=\eta x+\phi_{0}(t)+\sum_{n=1}^{N}\left[\phi_{n}(t) \cos \left(\frac{n \pi x}{l}\right)\right. \\
\left.+\psi_{n}(t) \sin \left(\frac{n \pi x}{l}\right)\right],
\end{array}
$$

where the $\phi_{n}(t)$ and $\psi_{n}(t)$ are unknown functions of time, $l$ is the normalized junction length, and $\eta$ is a constant chosen to take appropriately into account the boundary conditions. For the annular geometry junction the boundary conditions are

$$
\phi(x, t)=\phi(x+l, t)+2 m \pi,
$$

where $m$ is the difference between the number of fluxons and antifluxons present along the junction (since the junction is a closed loop $m$ is a conserved quantity). In this case,

$$
\eta=\frac{2 \pi m}{l}
$$

For the overlap geometry junction the boundary conditions are

$$
\phi_{x}(0, t)=\phi_{x}(l, t)=\eta
$$

where now $\eta$ represents the normalized value of the external magnetic field along the $y$ direction in the junction plane. Moreover, the boundary conditions [Eq. (5)] impose that $\psi_{n}=0$ for all $n$. All the results obtained herein are referred to the case where no external magnetic field is applied.

Although other geometrical configurations are possible, only the above two have been considered because they are the most useful for the understanding of the junction dynamics.

Inserting Eq. (2) into Eq. (1) and using the orthogonality of the trigonometric functions, the following set of differential equations is obtained:

$$
\begin{aligned}
& \ddot{\phi}_{0}+\mu_{0} \dot{\phi}_{0}-\gamma=-\frac{1}{l} \int_{0}^{l} \sin \phi d x, \\
& \ddot{\phi}_{n}+\mu_{n} \dot{\phi}_{n}+\omega_{n}^{2} \phi_{n}=-\frac{2}{l} \int_{0}^{l} \sin \phi \cos \left[\frac{n \pi x}{l}\right] d x, \\
& n=1,2, \ldots, N, \\
& \ddot{\psi}_{n}+\mu_{n} \dot{\psi}_{n}+\omega_{n}^{2} \psi_{n}=-\frac{2}{l} \int_{0}^{l} \sin \phi \sin \left(\frac{n \pi x}{l}\right) d x, \\
& n=1,2, \ldots, N .
\end{aligned}
$$

Here $\omega_{n}=(n \pi / l), \mu_{n}=\alpha+\omega_{n}^{2} \beta$ and overhead dots denote time derivatives.

The set of equations (6) is solved using a sixth-order predictor-corrector method with variable step size, ${ }^{10}$ while the right-hand sides of Eqs. (6) are evaluated by fast-Fourier-transform routine. ${ }^{11}$ The accuracy of the numerical integration was checked by decreasing the time step and by increasing the number of spatial harmonic components considered. A typical value for the time step was $\Delta t=0.05$ while a choice of a number of spatial harmonics equal to twice the junction length was always appropriate.

The other numerical scheme employed was an implicit finite difference method ${ }^{12}$ where the phase $\phi$ is restricted to a square mesh

$$
\phi_{p}^{q} \equiv \phi(p \Delta x, q \Delta t) .
$$

The derivatives in Eq. (1) are approximated by a secondorder Taylor expansion in the step size, with a time average over one time step for the $x$ derivatives and inserted into Eq. (1). The boundary conditions are handled by introducing virtual extra points at the edge of the mesh in the usual way. A predictor-corrector loop is used to evaluate the nonlinear term in Eq. (1), enhancing in this way the stability of the whole scheme. Finally a set of linear algebraic equations with a tridiagonal coefficient matrix is obtained in the form

$$
\underline{A} \mathbf{x}=\mathbf{y},
$$

where

$$
\mathbf{x}=\left(\phi^{q+1}, \ldots, \phi_{N}^{q+1}\right)
$$

and $\mathbf{y}$ is an appropriate function of the phase values at the mesh points and previous two time steps. Equation (8) is solved by the "double-sweep method"12 which has been demonstrated to be stable as long as the matrix $\underline{A}$ is diagonally dominant, i.e., in this case

$$
\frac{3(\beta+\Delta t)}{\alpha(\Delta x)^{2}+2(\Delta x)^{2} / \Delta t+2 \beta+2 \Delta t}<1 .
$$

Again the accuracy of the results has been checked by halving both the space step $\Delta x$ and the time step $\Delta t$. Although it may seem exaggerated to use two different numerical methods to solve the same equation, this was very useful to test the independence of the numerical results on the scheme used. This fact is very important when dealing with dynamical states parametrically driven to unstable regions. Another point to consider is that, al- 


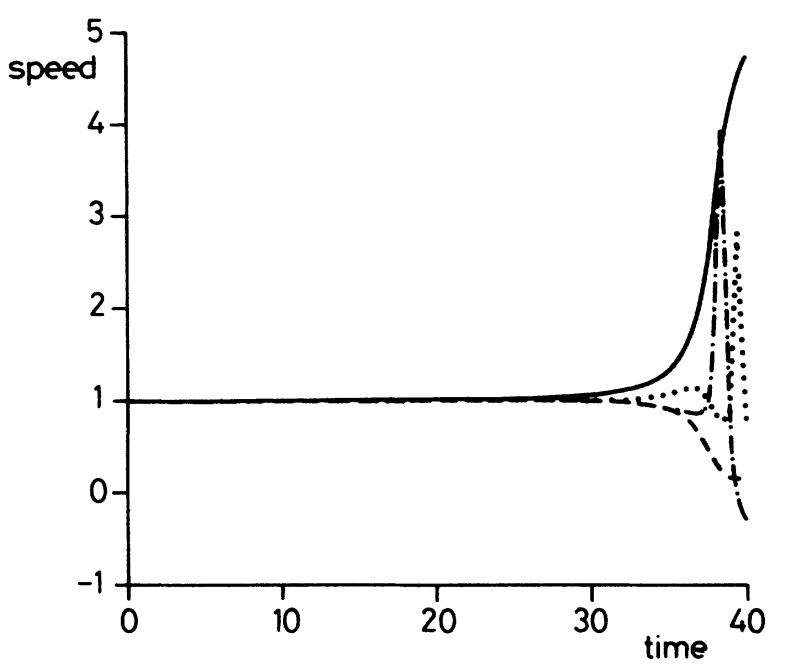

FIG. 1. Time evolution of the first four mode speeds. The modes considered are the 0th (solid curve), the 1st (dashed curve), the 2 nd (dash-dotted curve), and the 3rd (dotted curve).

though the finite difference method seems to be more efficient for long junctions, the multimode method is able to give more physical information about the dynamical states investigated. For example, looking at the phase velocity of each mode in annular junctions is very useful to determine whether or not stationary motion conditions are achieved. The mode phase velocity is defined as

$$
u_{n}=-\frac{F_{n}\left(\phi_{t}\right)}{F_{n}\left(\phi_{x}\right)},
$$

where $F_{n}(Y)$ is the $n$th component of the spatial Fourier transform of $Y$. This definition is based on the idea that each Fourier component of the phase travels with a modulating velocity $u_{n}$ which is slowly varying on the time scale of the time step used in the integration scheme. In Fig. 1 a plot of the time dependence of the phase velocity of the first four modes is shown for the case of an annular geometry junction with $l=16, \alpha=0.18, \beta=0.01$, $\gamma=0.89, m=1$. The parameter values correspond to the top of the first current singularity and the time evolution shows how the fluxon solution becomes unstable and breaks down at the time $t \sim 35$. In fact, as long as the fluxon is stable and travels along the junction, all the modes have the same velocity, the fluxon velocity, whereas when the solution becomes unstable it undergoes a transitory state where all the modes behave differently and finally reaches a new stable configuration where again all the modes have the same velocity (not shown in the figure).

\section{ANNULAR GEOMETRY}

In this section a study of the mechanism for the switching from the "fluxon" state (ZFS1) to the "rotating" state (McCumber branch) is carried out for junctions of annular geometry. The choice of the annular geometry is made because in such a system there are no collisions

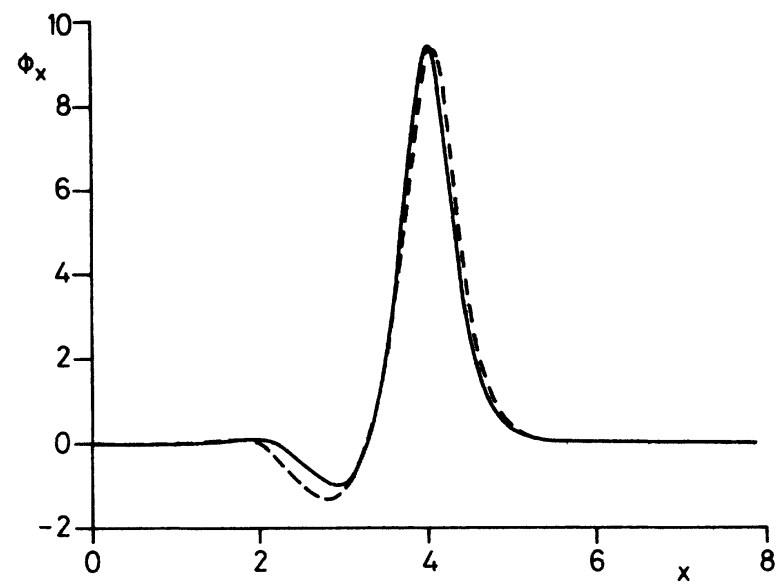

FIG. 2. $\phi_{x}$ at two different bias levels: $\gamma=0.75$ (solid curve) and $\gamma=0.8$ (dashed curve).

of the fluxons with the boundaries; thus the resulting dynamics is smoother. Moreover, since junction normalized lengths $l \gg 1$ are always considered, the motion of a single fluxon on an infinite Josephson transmission line is well approximated. This allows a reduction of Eq. (1) to a third-order ordinary differential equation (ODE) for the traveling phase profile, reducing the problem to the study of a low-dimensional system.

In Figs. 2-4 the initial condition of the fluxon line shape proposed by Ferrigno and Pace $^{5}$ is used,

$$
\begin{aligned}
\phi_{0}(x, t)= & \sin ^{-1} \gamma \\
+4 \tan ^{-1}\{\exp [ & \left(x-u_{0} t\right) \\
& \left.\left.\quad \times\left(1-\gamma^{2}\right)^{1 / 4} /\left(1-u_{0}^{2}\right)^{1 / 2}\right]\right\},
\end{aligned}
$$

where $u_{0}$ is computed from power-balance considerations $^{3}$

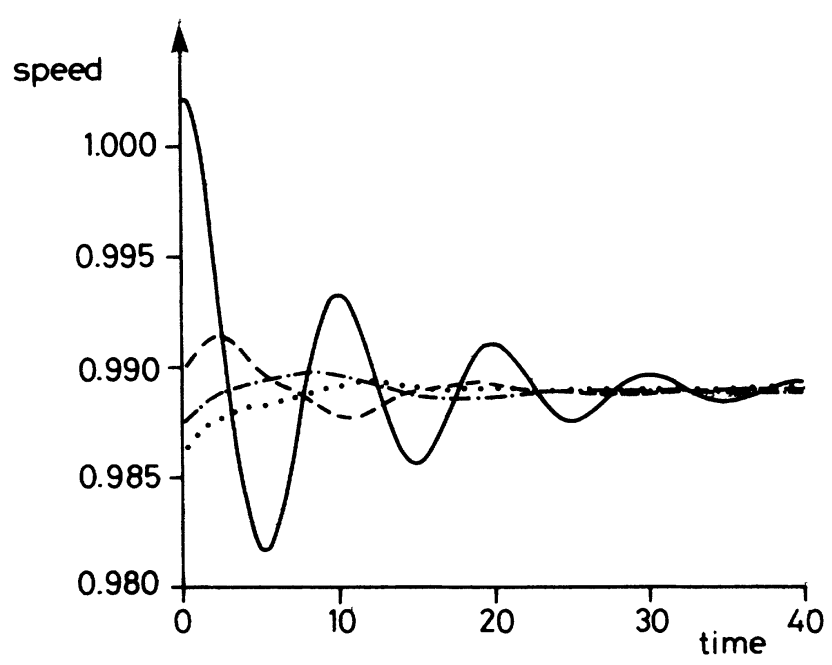

FIG. 3. Stabilization of the fluxon after a bias change illustrated by the first four mode speeds as in Fig. 1. $\alpha=0.18$, $\beta=0.1, \gamma=0.85$, and $l=16$. 


$$
\gamma=\frac{4 u_{0}}{\pi} \frac{\left(1-\gamma^{2}\right)^{1 / 4}}{\left(1-u_{0}^{2}\right)^{1 / 2}}\left[\alpha+\frac{\beta}{3} \frac{\left(1-\gamma^{2}\right)^{1 / 2}}{1-u_{0}^{2}}\right]
$$

In the limit $\gamma \rightarrow 0, u_{0}$ assumes the value found by McLaughlin and Scott. ${ }^{3}$ After some time the solution relaxes toward a fixed profile traveling with a constant speed $u$. The value of $u$ is determined either by a measurement of the mode phase velocities, or by a direct measurement of the fluxon position, depending on the

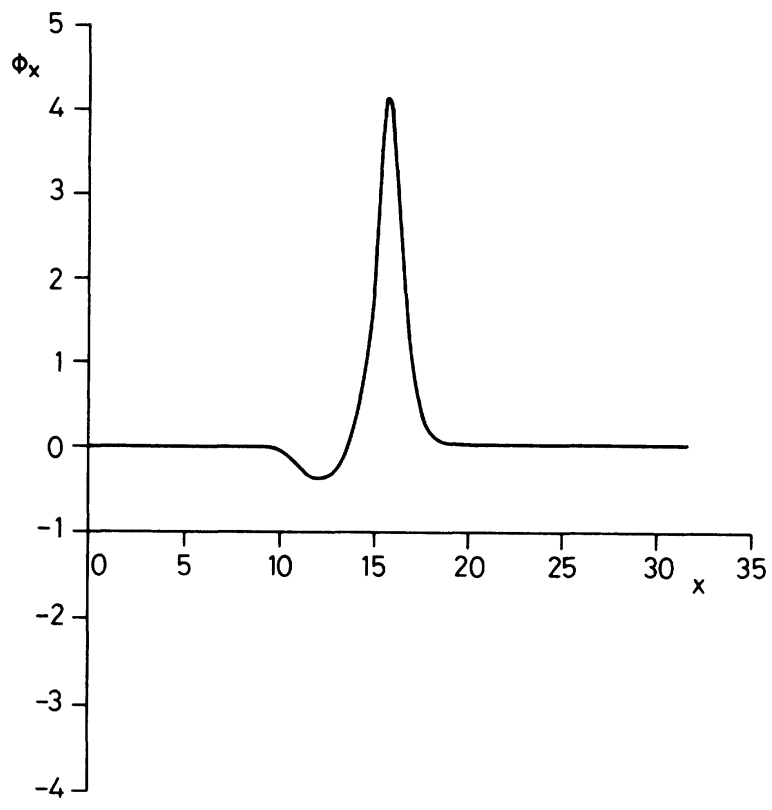

(a)

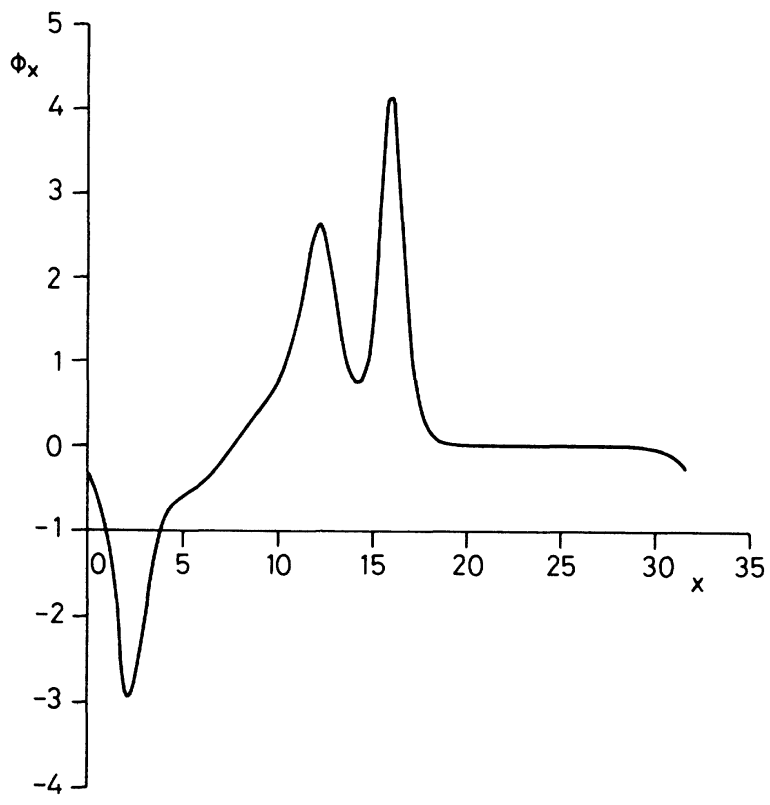

(c) particular numerical code used.

When the $\beta$ losses are absent, ${ }^{13}$ a constant speed $u$ is found for all values of $\gamma$ less than one. If a small $\beta$ loss is present, i.e., $\beta \ll \alpha$, a constant speed $u$ is not found for $\gamma$ values very close to one. For $\gamma>1$ there are no more static solutions of Eq. (1) therefore, a solution of fluxon structure is not possible. This leads to the first kind of switching mechanism. It can be described as the disappearing of the stable equilibrium solutions of Eq. (1) which form the asymptotic state of any fluxonlike solu-

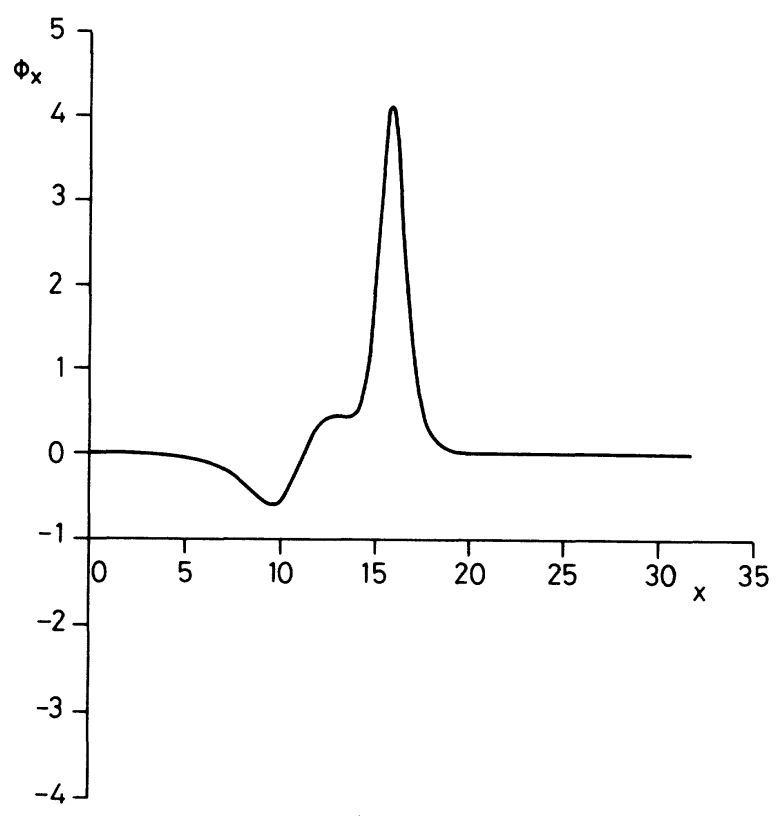

(b)

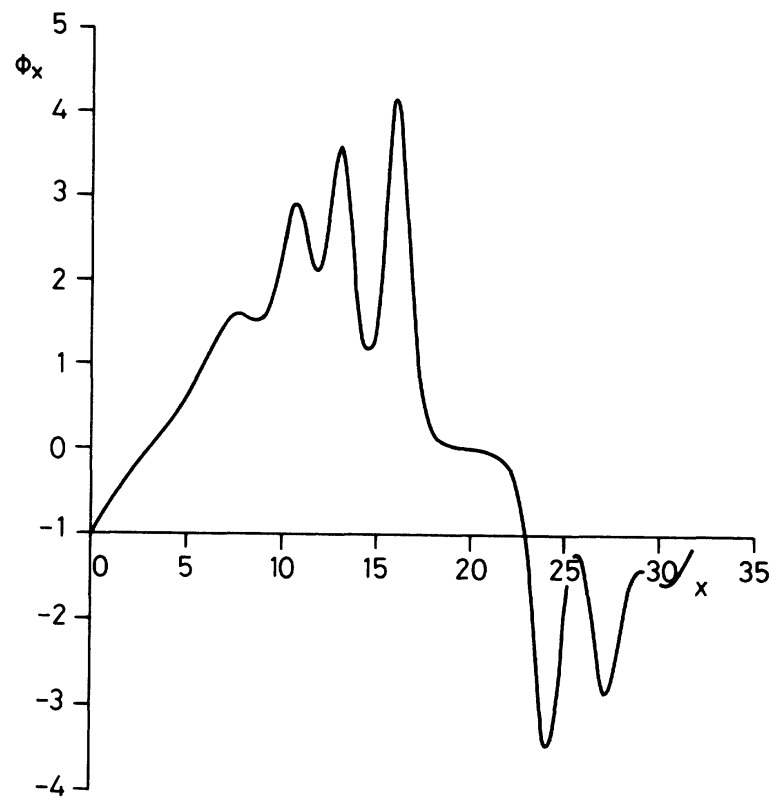

(d)

FIG. 4. Time sequence of the switching. (a) $t=25$; (b) $t=50$; (c) $t=55$; (d) $t=60$; (e) $t=65$; (f) $t=70$; (g) $t=75$. 


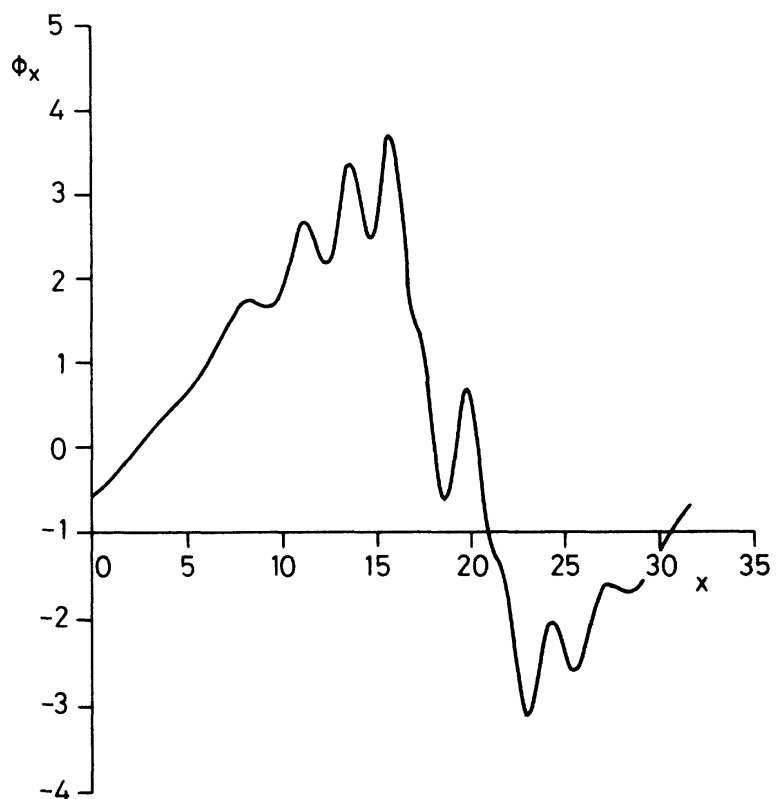

(e)

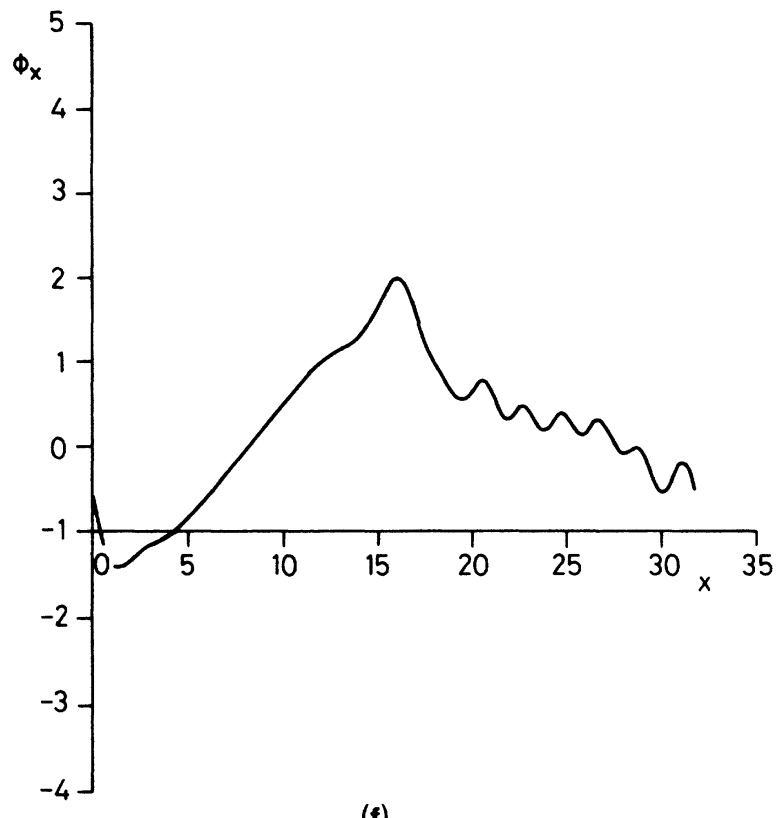

(f)

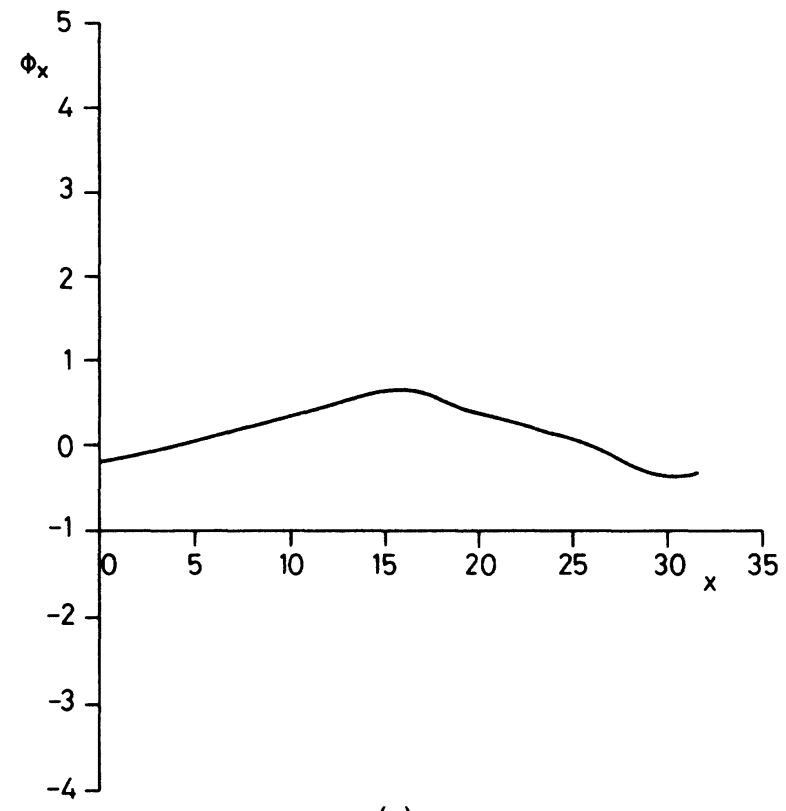

(g)

FIG. 4. (Continued).

tion. In terms of the chain of pendula analog, this correspond to the starting of a uniform rotation of all the pendula. If Ref. 14, Burkov and Lifsic have made a detailed stability analysis of Eq. (1) with $\beta=0$ and with periodic boundary conditions corresponding to an annular geometry junction. Their results also reveal stability of the fluxon when $\gamma<1$. The same result is found in Refs. 15 and 16 for a fluxon in an interval extending form $-\infty$ to $+\infty$. However, the stability analyses in Refs. 14 and 17 are valid only when the $\beta$ losses are absent. We shall show in the following that the effect of the $\beta$ losses is to decrease the critical value of $\gamma$ for which a stable fluxon motion can occur.
The presence of the perturbative terms in Eq. (1) introduces a modification of the fluxon line shape from the sine-Gordon one. ${ }^{4}$ The modification becomes more pronounced for increasing bias values. In Fig. 2 the numerical results for the $x$ derivative of the fluxon line shape are shown. The parameters are: $\alpha=0.05, \beta=0.02, l=8$, $\gamma=0.75$ (solid curve), and $\gamma=0.8$ (dashed curve). The main difference from the sine-Gordon form of the fluxon line shape in Fig. 2 is the presence of an overshoot at the trailing edge of the fluxon. The overshoot is present when the surface impedance term $\beta$ is not negligible and is more pronounced at high bias. In the following we will show that it is just the presence of the $\beta$ term in Eq. (1) 
that limits the maximum bias current that sustains the fluxon motion in annular junctions.

In order to determine the critical current values for the switching, the following numerical procedure has been followed. At the beginning, Eq. (12) is used as initial condition with a value of $\gamma$ relatively small (in order to be in a stable region). After some time of integration, $\gamma$ is increased slowly (with a time derivative $<10^{-3}$ ) to a new value. Then Eq. (1) is integrated for some time (typically 50 units) at constant $\gamma$ to allow a stabilization of the solution. The whole procedure is repeated again until a switching is observed. The measure of the mode phase velocities is used to check that the fluxon has assumed a stable profile. In Fig. 3 is shown the stabilization process in terms of the time evolution of the speed of the first four modes. As is clearly shown, all the modes tend rapidly to have the same speed, indicating that the fluxon has assumed a constant profile. When the critical value $\gamma_{\text {sw }}$ is reached, the stabilization of the modes speed does not take place and a switching is observed.

In Figs. 4(a)-4(g) a detailed time sequence of the switching is shown. The parameters are, in this case, $\alpha=0.18, \beta=0.1, l=32$, and $0.89 \leq \gamma \leq 0.9$. All the plots are referred to a reference frame moving with the fluxon. The switching can be described in the following way: First the overshoot at the trailing edge of the fluxon starts to grow in size and decreases its speed [Figs. 4(a)-4(b)]; when the overshoot is large enough, it breaks in a fluxon-antifluxon pair, Fig. 4(b) (in this plot a fluxon is represented by a positive pulse and an antifluxon by a negative one); the new fluxon starts to move forward and bunches with the original one, while the new antifluxon starts to move backward, driven by the current bias [Fig. 4(c)]; the process of nucleation of fluxon-antifluxon pairs continues adding new fluxons and antifluxons [Fig. 4(d)]; when the group of fluxons meets the group of antifluxons which has traveled all the way around the junction, a multiple collision occurs [Figs. 4(e) and 4(f)]. These finding were briefly reported in Ref. 17. As a result a net energy loss occurs in each fluxon (or antifluxon) to such an extent that they are not able to survive the next collision and form breatherlike structures; these structures do not gain energy from the bias and relax down to the flat configuration: $\phi_{x}=2 \pi / l, \phi_{t} \gg 1$ [Fig. 4(g)]. The final state obtained (a uniform phase twist increasing quickly in time) corresponds to the McCumber curve for the annular junction.

The mechanism for the switching just described suggests that the presence of the overshoot in the fluxon line shape plays a vital role for the triggering of the switching. The qualitative behavior of the overshoot can be understood by studying the traveling wave reduction of Eq. (1). With the following change of variables

$$
\xi=x+u t
$$

Eq. (1) reduces to

$$
\left(1-u^{2}\right) \phi_{\xi \xi}-\sin \phi=\alpha u \phi_{\xi}-\beta u \phi_{\xi \xi \xi}-\gamma .
$$

This ODE has a three-dimensional phase space with fixed points $\phi_{j}=\sin ^{-1} \gamma \pm 2 j \pi, \quad \phi_{\xi, j}=0, \quad \phi_{\xi \xi, j}=0$,

$\phi_{k}=\pi-\sin ^{-1} \gamma \pm 2 k \pi, \quad \phi_{\xi, k}=0, \phi_{\xi \xi, k}=0$.

The single fluxon solution of Eq. (1) corresponds to a curve connecting the points $\phi_{j}$ and $\phi_{j+1}$, as is shown in Fig. 5. Since the overshoot occurs near the $\phi_{j}$ point, a linearization of Eq. (15) around this fixed point can provide useful information on the character of the oscillations forming the overshoot. Linearizing Eq. (15) around $\phi_{j}$ gives

$$
\left(1-u^{2}\right) y_{\xi \xi}-\left(1-\gamma^{2}\right)^{1 / 2} y=\alpha u y_{\xi}-\beta u y_{\xi \xi \xi},
$$

where $\|y\| \ll 1$. This equation has a general solution of the form

$$
y=A e^{\lambda_{1} \xi}+B e^{\lambda_{2} \xi}+C e^{\lambda_{3} \xi},
$$

where $A, B$, and $C$ are integration constants and $\lambda_{1}, \lambda_{2}$, and $\lambda_{3}$ are the roots of the characteristic polynomial:

$$
P(\lambda)=-\lambda^{3}-\frac{1-u^{2}}{\beta u} \lambda^{2}+\frac{\alpha}{\beta} \lambda+\frac{\left(1-\gamma^{2}\right)^{1 / 2}}{\beta u} .
$$

Because of its structure, $P(\lambda)$ has always a positive real root and can have either two negative real roots or two complex conjugate roots with negative real part. This implies that the fixed points are either saddle points or saddle foci, as described by Hayashi. ${ }^{18}$ Equation (19) can be solved by standard methods, obtaining a fairly complicated expression for $\lambda_{1}, \lambda_{2}$, and $\lambda_{3}$. It should be noted, however, that the solution of Eq. (19) has the speed $u$ as an independent parameter, while $u$ is in reality a function of the other parameters $\alpha, \beta$, and $\gamma$. Since the exact relation for $u$ is not known, only approximate results can be then obtained by this linear analysis. Assuming, for high bias, $u=1$, it is easy to compute that in order to have complex roots, the bias must satisfy the following condition:

$$
\gamma<\left(1-\frac{4}{27} \frac{\alpha^{3}}{\beta}\right)^{1 / 2}
$$

Since, for reasonable values of $\alpha$ and $\beta$, the right-hand side of Eq. (20) is always very close to one, this condition states that the oscillating overshoot, associated with the complex roots of Eq. (19), is always present. In the same

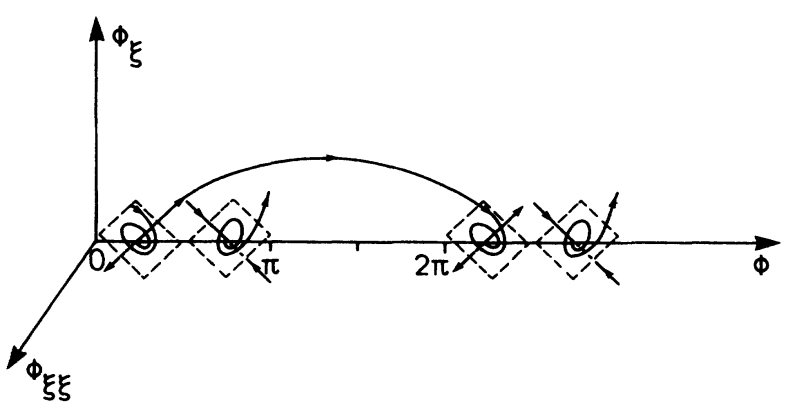

FIG. 5. Sketch of the phase space for Eq. (15). The fluxon solution connecting $\phi_{j}$ to $\phi_{j+1}$ is shown. 
way it is possible to compute the period $T$ and the decay rate $\mu$ of the overshoot oscillations as ${ }^{4}$

$$
\begin{aligned}
& T=\frac{\pi}{\sqrt{3}} \frac{\beta^{1 / 3}}{\left(1-\gamma^{2}\right)^{1 / 6}}, \\
& \mu=-\frac{1}{2} \frac{\left(1-\gamma^{2}\right)^{1 / 6}}{\beta^{1 / 3}},
\end{aligned}
$$

where terms of the order of $\alpha^{3} / \beta$ have been neglected. The result from Eqs. (21) and (22) has been checked with the numerical results, finding good agreement. However, because of the limitation mentioned above, this phasespace analysis cannot predict the critical bias current value at which the fluxon solution becomes unstable. A qualitative explanation of the switching is nevertheless possible. Perhaps a careful global analysis of the ODE, Eq. (15), may lead to a quantitative description of the onset of the switching.

\section{OVERLAP GEOMETRY}

In this section a study of the mechanism for the switching in junctions of overlap geometry is presented. Overlap geometry Josephson junctions with normalized lengths $l \gg 1$ and without external magnetic field applied, i.e., $\eta=0$, are considered. In Fig. 6 an experimental current-voltage characteristic is shown, with six ZFS's. The first zero-field step (ZFS1) results from the oscillation of one fluxon; the next step (ZFS2) corresponds to two oscillating fluxons, etc. For all steps, at sufficiently high bias current, the fluxon oscillations become unstable and a switch to the McCumber curve occurs. The switching is indicated by the arrows in Fig. 6. Figure 7 depicts the phase $\phi(x, t)$ of one fluxon performing a stable oscillation back and forth at $\gamma=0.5$, as obtained from Eq. (1) solved numerically. The parameter values used are $\alpha=0.05, \beta=0.02$, and $l=8$. An overshoot behind the fluxon is clearly visible. Increasing $\gamma$ to 0.7 the fluxon becomes unstable and a fast spatially uniform rotation emerges after a short transient. In Fig. 8 this transient motion is shown in more detail for a junction of length $l=12$ and with $\gamma=0.74$ using otherwise the same parameter values as in Fig. 7. Figure 8 displays

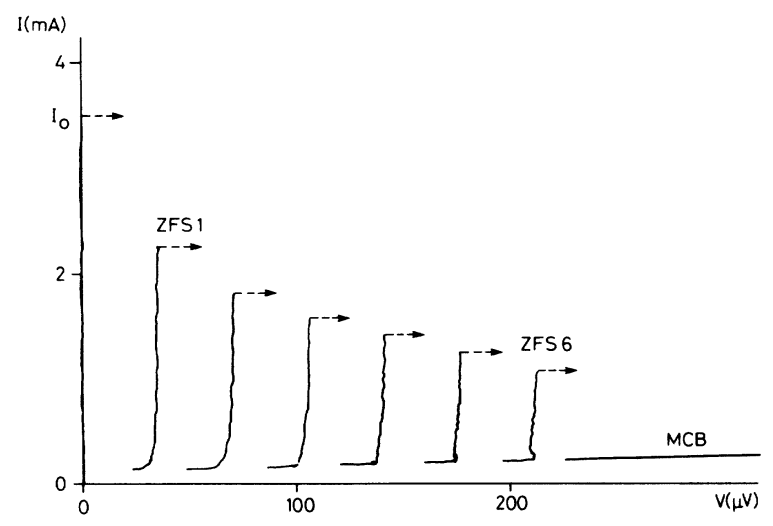

FIG. 6. Experimental $I-V$ characteristic of a long Josephson junction of overlap geometry. Six ZFS's are shown.

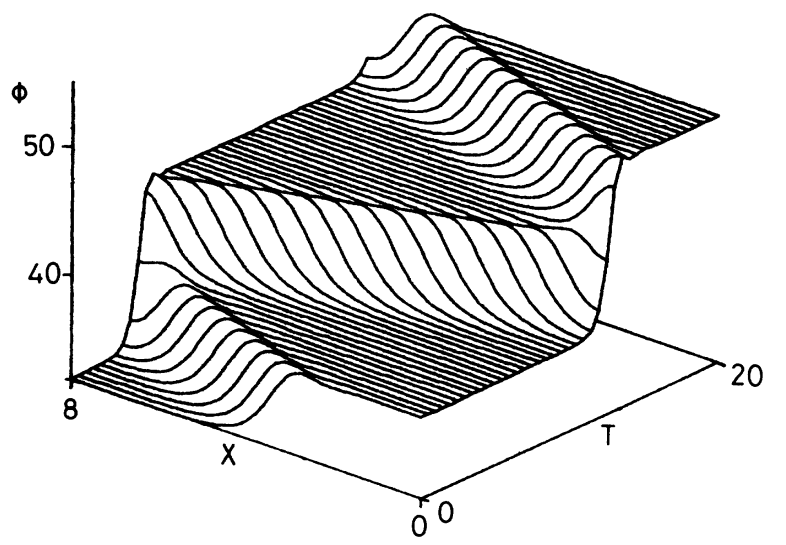

FIG. 7. Stable fluxon oscillations corresponding to ZFS1 in an overlap junction.

the phase at successive times $t$ during the switching starting at the arbitrarily chosen reference time $t=0$. At $\gamma=0.73$ the fluxon oscillation is stable. Increasing $\gamma$ to the value 0.74 the fluxon becomes unstable and after a couple of oscillations the fluxon hits the right-hand boundary $(l=12)$ at time $t \sim 6$ and during this reflection it becomes unstable. Immediately after the reflection the phase has increased by $4 \pi$ but is now unstable and it continues increasing after the time $t \sim 14$ leading to the formation of additional fluxons which travel from the right-

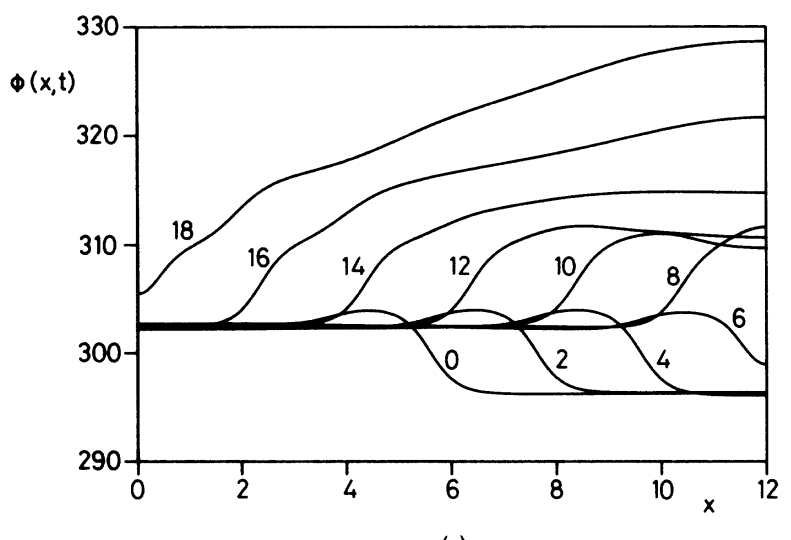

(a)

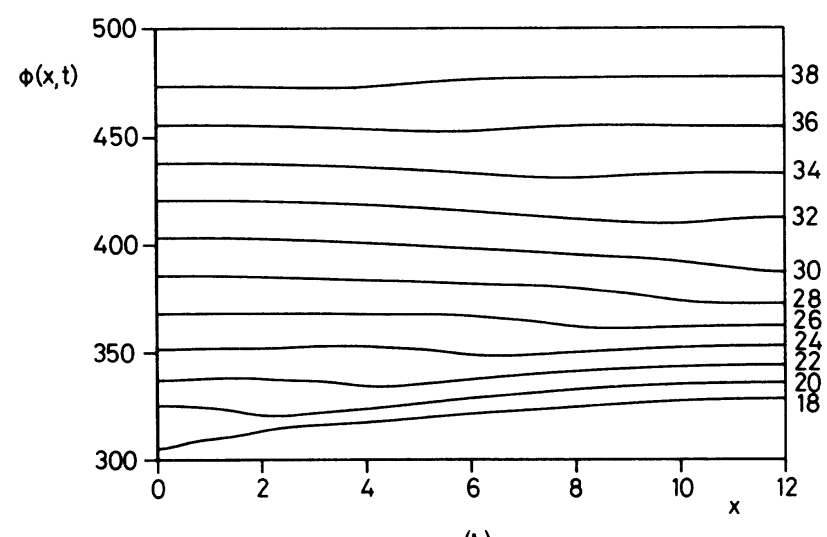

(b)

FIG. 8. Time sequence of the switching. (a) $0 \leq t \leq 18$. (b) $18 \leq t \leq 38$. 
hand boundary towards the left-hand boundary. At time $t \sim 18$ these fluxons are reflected from the left-hand boundary and annihilate the incoming ones. Eventually the phase develops into the spatially uniform rotating solution as can be seen from Fig. 8(b). From Fig. 8 it is evident that the fluxon is destabilized at one of the boundaries during a reflection and thereby triggers the formation of successive fluxons which unwind during the next reflection at the opposite boundary. The same finding has been reported by Cirillo et al. ${ }^{19}$ from a study of the mechanical analog of the long Josephson junction.

The switching can be illustrated also in terms of energy. The sine-Gordon energy ${ }^{3}$ of the junction is

$$
H_{T}=\int_{0}^{l}\left[\frac{1}{2}\left(\phi_{x}\right)^{2}+\frac{1}{2}\left(\phi_{t}\right)^{2}+1-\cos \phi\right] d x .
$$

In terms of the pendula model, the first term in Eq. (23) is the elastic energy arising from the elastic coupling between the pendula, the second term is the kinetic energy, and finally the term in $(1-\cos \phi)$ is the potential energy measured from the static downward equilibrium state. In the case where the dissipation and the external bias are absent, Eq. (1) reduces to the pure sine-Gordon equation which can be written as a Hamiltonian system with the Hamiltonian given by Eq. (23). When the perturbative terms are present, $H_{T}$ is merely the total energy of the system. By differentiating Eq. (23) with respect to the
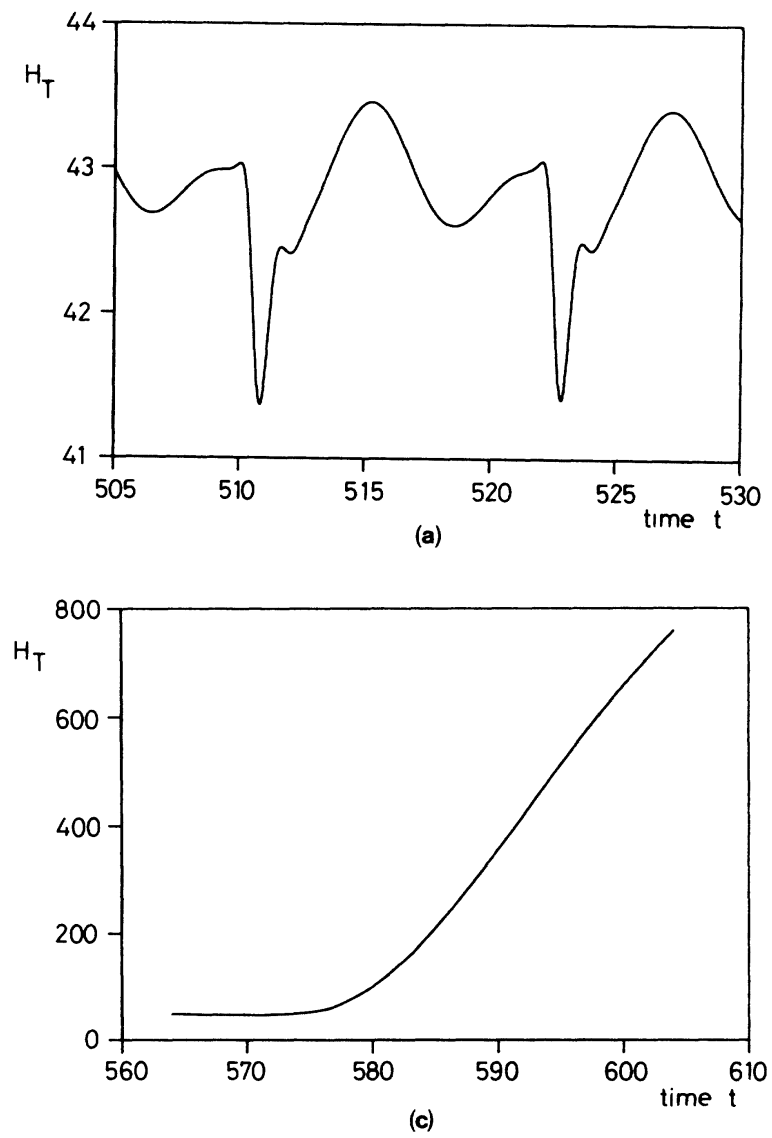

FIG. 9. Time evolution of (a) the total energy $H_{T}$, and (b) the powers $P_{\gamma} P_{\alpha}$, and $P_{\beta}$ during the stable motion on ZFS1. Time evolution of (c) the total energy $H_{T}$, and (d) the powers $P_{\gamma}, P_{\alpha}$, and $P_{\beta}$ during the switching from ZFS1. time $t$ and using Eq. (1) together with the boundary conditions, Eq. (5), it is easy to obtain

$$
\frac{d H_{T}}{d t}=P_{\gamma}+P_{\alpha}+P_{\beta}+\eta\left[\phi_{t}(l, t)-\phi_{t}(0, t)\right]
$$

where the power input and output have been defined according to

$$
\begin{aligned}
& P_{\gamma}=+\int_{0}^{l} \gamma \phi_{t} d x, \\
& P_{\alpha}=-\int_{0}^{l} \alpha\left(\phi_{t}\right)^{2} d x, \\
& P_{\beta}=-\int_{0}^{l} \beta\left(\phi_{x t}\right)^{2} d x .
\end{aligned}
$$

Note that the terms $P_{\alpha}$ and $P_{\beta}$ extract energy from fluxons or antifluxons whereas the term $P_{\gamma}$ is an energyinjection term which accelerates fluxons and antifluxons in opposite directions.

Figure 9 shows the time evolution of the total energy and the power input and output terms in Eqs. (25). Using the parameter values $\alpha=0.05, \beta=0.02$, and $l=12$, Figs. 9(a) and 9(b) depict the total energy and powers in the case of a stable fluxon oscillation at $\gamma=0.73$ just below the critical bias current $\gamma_{\mathrm{sw}}$. Over one period of oscillation the total energy remains constant and the power input balances the power output. During a reflection at one of the boundaries a fast $4 \pi$ change occurs in the

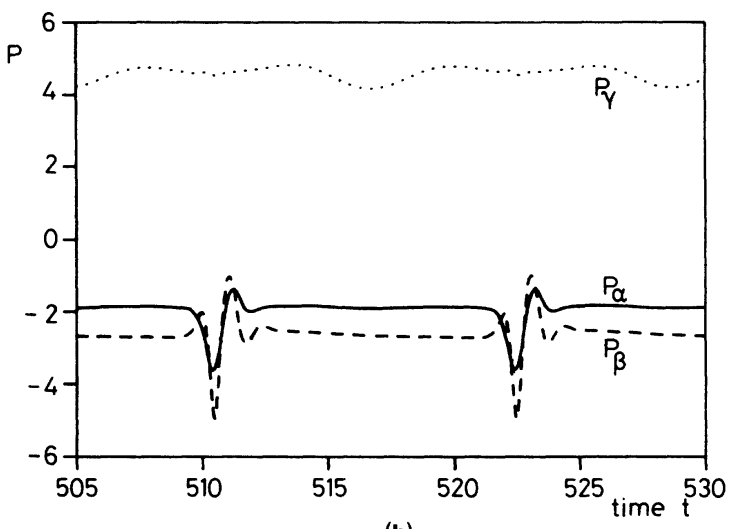

(b)

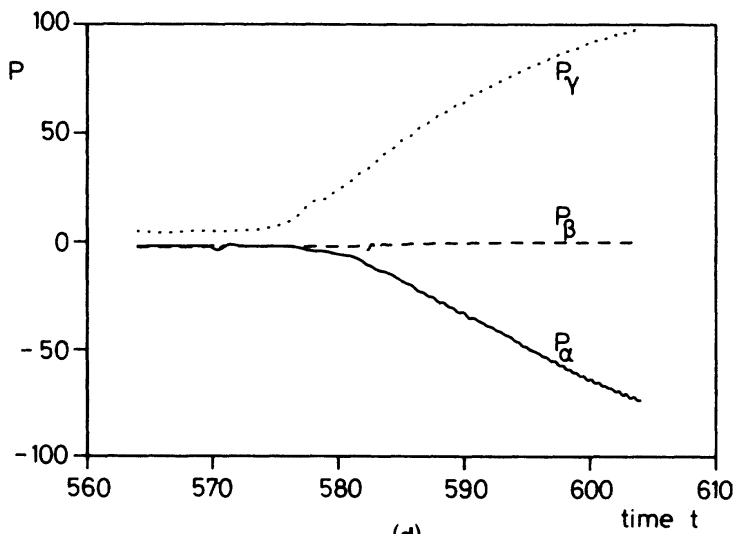

(d) 
phase $\phi$ and the dissipation from both terms in $\alpha$ and $\beta$ increases. This results in a pronounced dip in the total energy during a reflection. It is important to note that this energy loss at the boundaries is purely dissipative and is not a result of radiation from the junction end. Such radiation is not accounted for in this model. In Figs. 9(c) and 9(d) $\gamma$ has been increased to the value 0.74 where switching occurs (see also Fig. 8). During the switching the total energy significantly increases, as does the loss due to the $\alpha$ term. Eventually the system approaches the spatially uniform McCumber state and accordingly the loss due to the $\beta$ term vanishes. When equilibrium has been reached on the McCumber solution the averaged total energy will again be constant and over one period of oscillation $P_{\gamma}$ will balance $P_{\alpha}$. This equilibrium state is not shown in Figs. 9(c) and 9(d).

\section{STABILITY ANALYSIS BY BOUNDARY MODEL}

In this section a boundary-based model is introduced to explain the switching from the ZFS's to the McCumber curve in the overlap geometry Josephson junctions. The phenomenon of the switching in overlap junctions appears to be related to some instability that takes place at the junction boundary. This was first pointed out by Cirillo et al. ${ }^{19,20}$ from observations on the mechanical analog of a Josephson junction. The same idea can be deduced by a careful analysis of Figs. 7 and 8. In fact in Fig. 8(a) it can be seen that the reflection of the fluxon that occurs at $t \sim 6$ does not leave the boundary in a static phase configuration $\left(\phi=\sin ^{-1} \gamma+2 m \pi\right)$ as occurs when stable fluxon oscillations are observed (Fig. 7). A way to analyze this phenomenon can be to study the dynamics of the phase at the junction boundary (say at $x=0$ ). In terms of the chain of pendula analog of the PSGE this corresponds to studying the dynamics of a single pendulum, namely the last of the chain, under the fluxon-antifluxon collision that describes the process of reflection of a fluxon at the junction boundary. Following this idea one can rewrite Eq. (1) as

$$
\phi_{t t}+\alpha \phi_{t}+\sin \phi=\gamma+\phi_{x x}+\beta \phi_{x x t} .
$$

This equation can be viewed as the equation of a single pendulum [the left-hand side of Eq. (26)] driven by an effective force [the right-hand side of Eq. (26)]. This effective force is made of the external bias $\gamma$ and the coupling to other pendula of the chain $\left(\phi_{x x}+\beta \phi_{x x t}\right)$. The problem here is that the effective force is not known, being dependent on the solution $\phi$ of the same equation. A reasonable approximation may, however, be given by the analytic expression for the fluxon-antifluxon collision solution of the sine-Gordon equation, which can be rewritten in a form that takes into account the perturbative terms in Eq. $(26)^{4}$ (Ref. 4 ) as

$\phi^{0}=\sin ^{-1} \gamma+4 \tan ^{-1}\left[\frac{1}{u} \sinh \left(\frac{u t}{\Gamma}\right) \operatorname{sech}\left(\frac{x}{\Gamma}\right)\right]$,

where $\Gamma$ is the corrected Lorentz factor introduced in Eq. (12) as

$$
\Gamma=\frac{\left(1-u^{2}\right)^{1 / 2}}{\left(1-\gamma^{2}\right)^{1 / 4}}
$$

and the velocity $u$ may be determined by the single fluxon power-balance expression of Eq. (13). Thus Eq. (26) becomes

$\phi_{t t}+\alpha \phi_{t}+\sin \phi=\gamma+\phi_{x x}^{0}(x=0)+\beta \phi_{x x t}^{0}(x=0)$,

where

$$
\phi_{x x}^{0}(x=0, t)=\frac{-4 u \Gamma^{-2} \sinh \left(u \Gamma^{-1} t\right)}{u^{2}+\sinh ^{2}\left(u \Gamma^{-1} t\right)}
$$

and

$$
\begin{aligned}
& \phi_{x x t}^{0}(x=0, t) \\
& =\frac{-4 u^{2} \Gamma^{-3} \cosh \left(u \Gamma^{-1} t\right)\left[u^{2}-\sinh ^{2}\left(u \Gamma^{-1} t\right)\right]}{\left[u^{2}+\sinh ^{2}\left(u \Gamma^{-1} t\right)\right]^{2}} .
\end{aligned}
$$

Equation (29) has a static solution $\phi=\sin ^{-1}(\gamma)$ as $t \rightarrow-\infty$ corresponding to the configuration of the $x=0$ pendulum in the mechanical model when the fluxon is still far away. When the incoming fluxon reaches the $x=0$ point $(t \rightarrow 0)$ the right-hand side of Eq. (29) changes very fast leading to a $4 \pi$ increase of the phase. After the fluxon is reflected $(t \rightarrow+\infty)$, the phase can either relax to the new equilibrium state $\phi=\sin ^{-1}(\gamma)+4 \pi$ or go to the rotating state corresponding to the McCumber solution of the simple pendulum. The first case will correspond to a "normal" reflection and to the stable dynamical state of the ZFS's, while the second case will correspond to the switching to the McCumber branch is shown in Figs. 8 and 9. The value of the bias $\gamma$ that corresponds to the transition from the first case to the second one will then be the one that determines the switching from the ZFS's to the McCumber branch. In Fig. 10 the time evolution is shown of $\phi$ and of $\phi_{t}$ for different $\gamma$ values (curves 1-5) obtained by a numerical integration of Eq. (29). It can

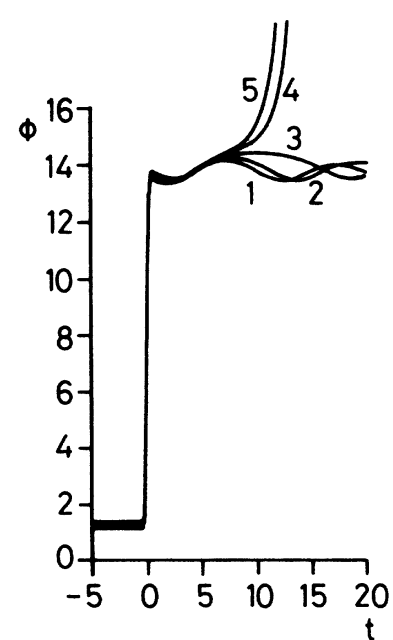

(a)

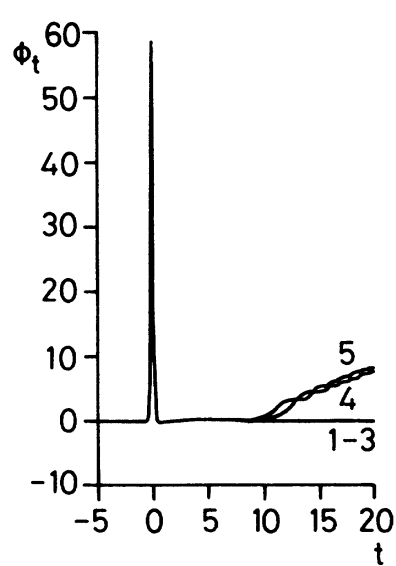

(b)
FIG. 10. Time evolution of $\phi$ (left) and $\phi_{t}$ (right) for the boundary model. The curves labeled 1-5 correspond to bias values from 0.90 to 0.98 in steps of 0.02 . The switching occurs at $\gamma=0.95 \pm 0.01$. $\alpha=0.05, \beta=0.02$, and $l=\infty$. 
clearly be seen that curve 3 (corresponding to $\gamma=0.94$ ) is the last stable state and a further increase of $\gamma$ leads to the switching.

\section{RESULTS AND DISCUSSIONS}

Our results are summarized in Fig. 11. Figure 11(a), curve $a$, shows the length dependence of the critical switching value of $\gamma$ for overlap junctions with $\alpha=0.05$ and $\beta=0.02$. Curve $b$ is the $l \rightarrow \infty$ limit for the switching value in annular junctions having the same loss parameters. As can be seen, the critical $\gamma$ for overlap junctions tends asymptotically to a value lower than that for annular junctions. In passing, we note that curve $a$ is quite similar in form to, but slightly higher in current than, the curve shown in Fig. 2 of Ref. 7 (nominally the same problem). In this connection, we comment that the value of $\gamma_{\text {sw }}$ obtained depends rather sensitively on how $\gamma$ is increased during the computations: the more "adiabatic" the increase, the more accurate the value of $\gamma_{\mathrm{sw}}$.

Figure 11(b) shows the $\alpha$ dependence, for $\beta=0.02$ and $l=12$, for overlap junctions (curve $a$ ), annular junctions (curve $b$ ), and for the boundary model (curve $c$ ). Once again, it is apparent that overlap junctions switch at lower current values than do annular junctions. Also, once again, our curve $a$ is quite similar to that shown in Fig. 4 of Ref. 7.

Figure 11(c) shows the $\beta$ dependence, for $\alpha=0.05$ and $l=12$, of $\gamma_{\mathrm{sw}}$ for the same three cases as before. As before, curve $a$ lies below curve $b$. Comparing our curve $a$, however, with the curve shown in Fig. 5 of Ref. 7, a significant difference is apparent: our result indicates that $\gamma_{\text {sw }}$ tends smoothly to 1 as $\beta \rightarrow 0$, whereas Zhang and Wu's curve bends over and terminates near $\gamma \simeq 0.7$. In this connection, we comment that the implicit finite difference routine of Ref. 12 , used also by Zhang and $\mathrm{Wu}$, was developed specifically to integrate Eq. (1) with $\beta \neq 0$. In fact, for $\beta=0$, Eq. (1) is a second- rather than thirdorder equation, and much more efficient explicit algorithms are available. ${ }^{21}$ Our experience indicates that for $\beta \rightarrow 0$ this implicit algorithm must be used with great care. Even though condition (10) is satisfied, unless very small spatial and temporal increments, $\Delta x$ and $\Delta t$, are used, the algorithm tends to generate spurious oscillations which can completely falsify results.

As is apparent from Figs. 11(b) and 11(c), the boundary model, curve $c$, gives a good qualitative description of the $\alpha$ and $\beta$ dependences of the computed $\gamma_{\text {sw }}$ values, curves $a$. The essential difference is an almost constant shift of about 0.15 in $\gamma$. This remarkable result strengthens the
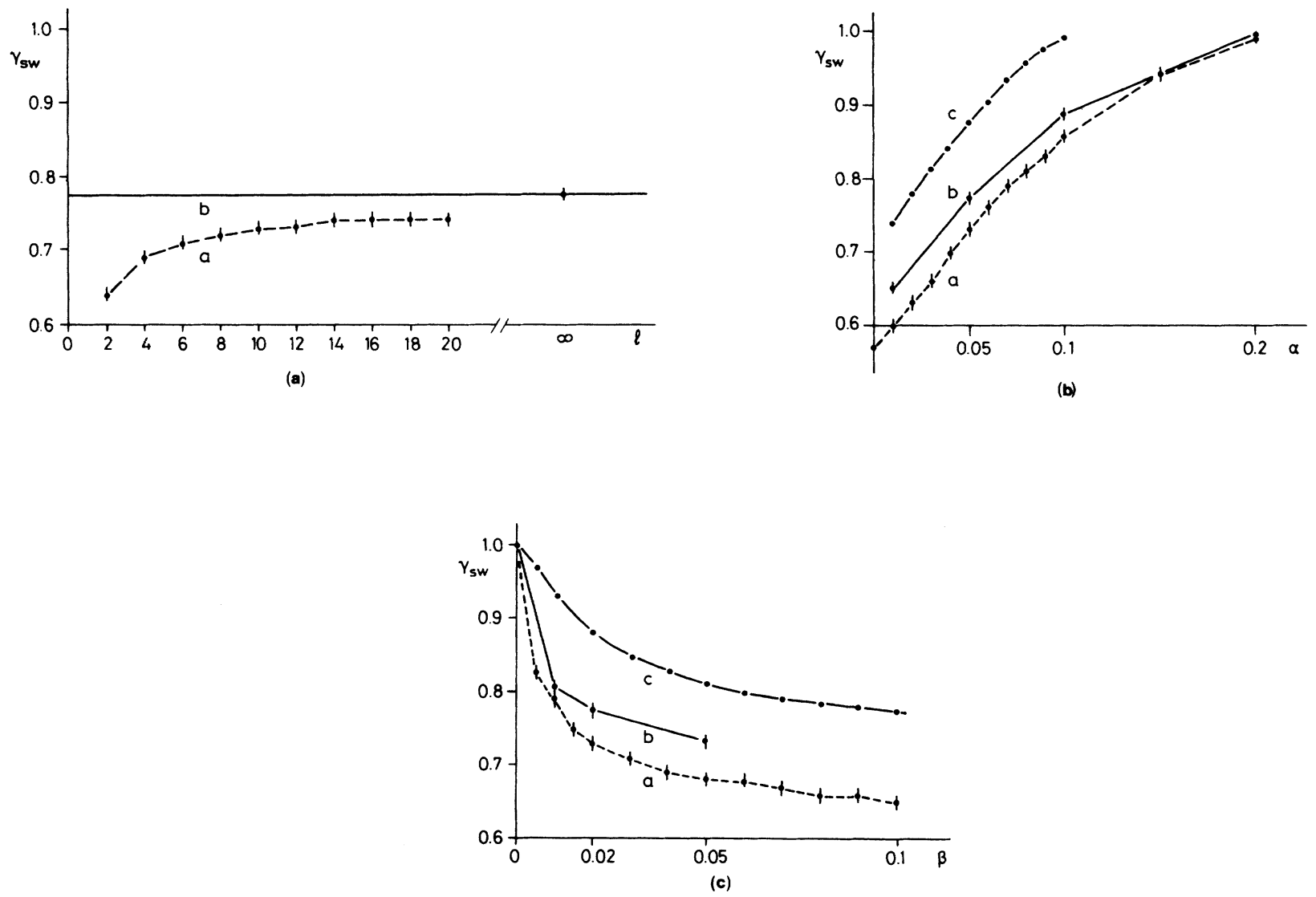

FIG. 11. (a) Dependence of the critical bias on the junction length. $a$ overlap geometry. $b$ annular geometry $(l \rightarrow \infty) . \alpha=0.05$ and $\beta=0.02$. (b) Dependence of the critical bias on $\alpha$. $a$ overlap geometry. $b$ annular geometry. $c$ boundary model. $\beta=0.02$ and $l=12$. (c) Dependence of the critical bias on $\beta$. $a$ overlap geometry $(l=12) . b$ annular geometry. $c$ boundary model. $\alpha=0.05$ and $l=12$. 
idea suggested by the numerical simulations that in long junctions of overlap geometry the switching phenomenon is connected to an instability that is generated at the boundaries after the fluxon-antifluxon reflection. The almost constant shift between the results of the simple model Eq. (29) and the ones from the complete model Eq. (26) is fully due to the choice (27) for $\phi^{0}$. In fact, as has been shown in the preceding sections, the sine-Gordon solutions are not anymore a good approximation to the solution of Eq. (26) at high bias values. Indeed, using in Eq. (29) a numerical solution of Eq. (26) for $\phi^{0}$ computed in a stable state close to the switching $(\alpha=0.05, \beta=0.02$, $\gamma=0.7, l=12)$ the correct switching value for $\gamma$ is computed $\left(\gamma_{\mathrm{sw}}=0.72\right)$.

\section{CONCLUSIONS}

In this paper we have addressed the problem of the stability of the fluxon motion at high bias values. Two different geometrical configurations have been considered. For the junctions of annular geometry the instability in the fluxon motion arises from the disappearance of the asymptotic static states for $\gamma \simeq 1$, in the case $\beta \ll 1$, or, for $\beta$ that are not small, from the nucleation of fluxon-antifluxon pairs. In the latter case the critical bias value is smaller than one and tends to decrease with increasing $\beta$ [Fig. 11(c)].

An analysis of the third-order ordinary differential equation (ODE) governing the traveling fluxons in the junction is able to describe qualitatively the deformation of the fluxon profile due to $\beta$ losses that are not small, but it cannot predict the critical bias value at which the fluxon solution becomes unstable.

In the overlap geometry junction the disappearing of the fluxon oscillations is due to an instability that is originated at the junction boundaries after a fluxonantifluxon reflection. This instability causes a switching to the McCumber curve for bias values lower than the ones required in the annular geometry case. However, the dependence of $\gamma_{\text {sw }}$ on the parameters $\alpha$ and $\beta$ is similar for the two geometries. The dependence of $\gamma_{\mathrm{sw}}$ on the junction length shows a saturation for $l \geq 10$, to a value smaller than the corresponding one in the annular junction case [Fig. 11(a)].

A simple model, based on the simulation of a fluxonantifluxon reflection, is able to reproduce qualitatively the numerical results. An analysis based on the Floquet theory supports the idea that the switching is due to a parametric excitation of the Fourier components of the phase. ${ }^{22}$ However, since all the components are involved in the process, it is not possible to obtain analytical expressions for the critical bias values.

\section{ACKNOWLEDGMENTS}

We thank M. Salerno for helpful discussions about the boundary model analysis. The financial support from the European Economic Community through Contract No. St-2-0267-J-C(A), from the European Research Office of the United States Army through Contract No. DAJA45-85-C-0042, the Thomas B. Thriges Fond (Denmark), and the Gruppo Nazionale di Struttura della Materia, Con- siglio Nazionale delle Ricerche-Centro Interuniversitario di Struttura della Materia, Ministero della Pubblica Istruzione (Italy) is gratefully acknowledged.
*Permanent address: Istituto di Cibernetica del Consiglio Nazionale delle Ricerche, via Toiano 6, I-80072 Arco Felice, Italy.

†Permanent address: Dipartimento di Fisica, Università degli Studi di Salerno, I-84100 Salerno, Italy.

${ }^{1}$ A. Barone and G. Paternó, Physics and Applications of the Josephson Effect (Wiley, New York, 1982).

${ }^{2}$ T. A. Fulton and R. C. Dynes, Solid State Commun. 12, 57 (1973).

${ }^{3}$ D. W. McLaughlin and A. C. Scott, Phys. Rev. A 18, 1652 (1978).

${ }^{4}$ S. Pagano, N. F. Pedersen, S. Sakai, and A. Davidson, IEEE Trans. Magn. Mag. 23, 1114 (1987).

${ }^{5}$ A. Ferrigno and S. Pace, Phys. Lett. 112A, 77 (1985).

${ }^{6}$ A. Davidson, B. Dueholm, B. Kryger, and N. F. Pedersen, Phys. Rev. Lett. 55, 2059 (1985).

${ }^{7}$ Y. M. Zhang and P. H. Wu, Extended Abstracts of the 1987 International Superconductivity Electronics Conference, Tokyo, Japan, 1987 (unpublished), p. 115.

${ }^{8}$ V. M. Vinokur, J. Phys. (Paris) 47, 1425 (1987).

${ }^{9}$ S. Pagano, M. P. Soerensen, R. D. Parmentier, P. L. Christiansen, O. Skovgaard, J. Mygind, N. F. Pedersen, and M. R. Samuelsen, Phys. Rev. B 33, 174 (1986).

${ }^{10}$ DGEAR routine, IMSL Inc., International Mathematical and Statistical Library (ed. 9), Houston, Texas, 1982.
${ }^{11}$ FFT2C routine, IMSL Inc., International Mathematical and Statistical Library (ed. 9), Houston, Texas, 1982.

${ }^{12}$ P. S. Lomdahl, O. H. Soerensen, and P. L. Christiansen, Phys. Rev. B 25, 5737 (1982); see also, P. S. Lomdahl, Ph.D. thesis, DCAMM, Lyngby Denmark, 1982 (unpublished).

${ }^{13}$ M. Büttiker and H. Thomas, Phys. Lett. 77A, 372 (1980); Phys. Rev. A 37, 235 (1988).

${ }^{14}$ S. E. Burkov and A. E. Lifsic, Wave Motion 5, 197 (1983).

${ }^{15}$ M. Büttiker and R. Landauer, Phys. Rev. A 23, 1397 (1981).

${ }^{16}$ K. K. Likarev, Dynamics of Josephson Junctions and Circuits (Gordon and Breach, New York, 1986).

${ }^{17}$ A. Davidson, N. F. Pedersen, and S. Pagano, Appl. Phys. Lett. 48, 1306 (1986).

${ }^{18}$ C. Hayashi, Nonlinear Oscillations in Physical Systems (McGraw-Hill, New York, 1964), p. 45.

${ }^{19}$ M. Cirillo, S. Pace, and B. Savo, in Proceedings of the 17th International Conference on Low Temperature Physics, edited by U. Eckern, A. Schmid, W. Weber, and H. Wühl (Elsevier, Amsterdam, 1984), Vol. 1, p. 703.

${ }^{20}$ M. Cirillo, R. D. Parmentier, and B. Savo, Physica 3D, 565 (1981).

${ }^{21}$ R. K. Dodd, J. C. Eilbeck, J. D. Gibbon, and H. C. Morris, Solitons and Nonlinear Wave Equations (Academic, London, 1982), Chap. 10.

${ }^{22}$ M. P. Soerensen (unpublished). 